\title{
Relationship between microstructure, stress and hardness in multilayer coatings
}

\author{
F.A. Burgmann", X.L. Xiao , D.G. McCulloch", D.R. McKenzie ${ }^{* *}$, M.M.M Bilek ${ }^{* *}$, B.K. Gan ${ }^{* *}$ and \\ L. Ryves* \\ *Applied Physics, School of Applied Sciences, City Campus, GPO Box 2476V, Melbourne 3001 \\ Victoria Australia \\ ${ }^{* *}$ Applied and Plasma Physics, School of Physics (A28), University of Sydney, Sydney 2006 NSW \\ Australia
}

The need to synthesize advanced materials which exhibit superior mechanical properties has increased over the past decade with the insurgence of machining and bio-medical devices into the global market. These applications including silicon-based semiconductor devices, Micro-ElectroMechanical Systems (MEMS), tool steel coatings and artificial organs require materials with a variety of properties including enhanced surface hardness and wear resistance, and reduced crack propagation and delamination. Significant advances in Physical Vapor Deposition (PVD), mainly Pulsed Filtered Cathodic Arc, have allowed accurate control of thin film properties enabling specifically tailored coatings to be engineered.

Much interest has been placed on multilayer thin film coatings which consist of alternating layers of different materials with structural features in the nanometer scale. Until now, published results have varied extensively, and debate over the existence of the Supermodulus and Supertoughness effects within multilayer coatings, consisting of brittle/soft layers, is largely widespread. These phenomena suggest an optimal bi-layer thickness at which a significant increase in hardness and toughness can be observed [1-3]. The abalone shell is one of nature's extraordinary examples of this effect, which is known to be a tough multilayer material made up of alternating soft/brittle layers [4]. The prospect of fabricating such nanostructured materials for practical applications is highly desirable. FIG. 1(a-b). show an image of the abalone shell and a cross sectional SEM micrograph of the multilayer structure respectively. By observing the microstructure of these materials, this project aims to explain these discrepancies and understand the relationship between the microstructure and properties in these materials, which currently is not well understood. In this work, we will compare stress and indentation hardness measurements of $\mathrm{Al} / \mathrm{W}$ and $\mathrm{Al} / \mathrm{AlN}$ multilayers with microstructure determined using cross-sectional transmission electron microscopy (X-TEM).

When producing thin film coatings by PVD techniques, deposition parameters including ion flux and deposition rate can be accurately controlled which are required when fabricating reproducible layer thicknesses [5]. Al/W multilayers will be synthesized using pulsed filtered cathodic arc PVD, and Al/ALN will be produced using a continuous DC arc, to a total thickness of $200 \mathrm{~nm}$, with varying bi-layer thicknesses. These multilayer systems were chosen as Al is a relatively soft material and W and ALN are relatively hard, which mimics the abalone shell. X-TEM is used to provide a detailed analysis of the samples including an accurate depth scale. X-TEM combines detailed structure and bonding information using electron diffraction and electron energy loss spectroscopy (EELS).

The Al/AlN multilayer samples show a maximum stress at a nominal bi-layer thickness of $15 \mathrm{~nm}$. These results suggest that films with a $15 \mathrm{~nm}$ bi-layer thickness are related to the maximum yield 
strength. The yield strength is thought to be related to the number of layers in the material. It is therefore expected that the stress will increase as the bi-layer thickness decreases since there are more hard/soft layers in a $200 \mathrm{~nm}$ coating at small bi-layer thicknesses. Therefore the decrease in stress at bi-layer thicknesses of less than $15 \mathrm{~nm}$ is somewhat unexpected. FIG. 2. shows the X-TEM images and Al maps produced from the Al plasmon peak of coatings with a nominal bi-layer thicknesses of $15 \mathrm{~nm}$. There is some evidence that the layered structure breaks down in this sample. The breakdown of the layered structure may explain the decrease in stress and hence yield strength of the coating for AL/ALN bi-layer thicknesses of less than $15 \mathrm{~nm}$.

\section{References}

[1] J.H. Xu et al., J. Appl. Phys. 89(7) (2001) 3674.

[2] N. Kikuchi et al., Surf. Coat. Technol. 126(2-3) (2000) 131.

[3] X. Chu et al., Surf. Coat. Technol. 61(1-3) (1993) 251.

[4] B.L. Smith et al., Nature, 399 (1999) 761.

[5] B.K. Gan et al., Plasma Sources Sci. Technol. 12 (2003) 508.

[6] This research was supported by Applied and Plasma Physics Department at the University of Sydney. The aid of Dr. Richard Tarrant, Dr. Bee Gan and Luke Ryves is gratefully acknowledged.
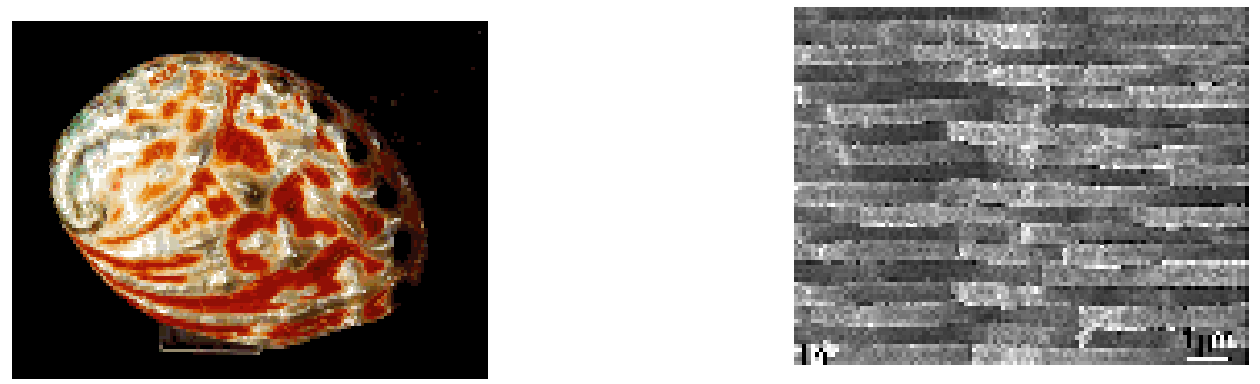

FIG. 1(a). : Optical image of an Abalone shell, and (b) cross-sectional SEM micrograph of multilayer stacks in an Abalone shell.
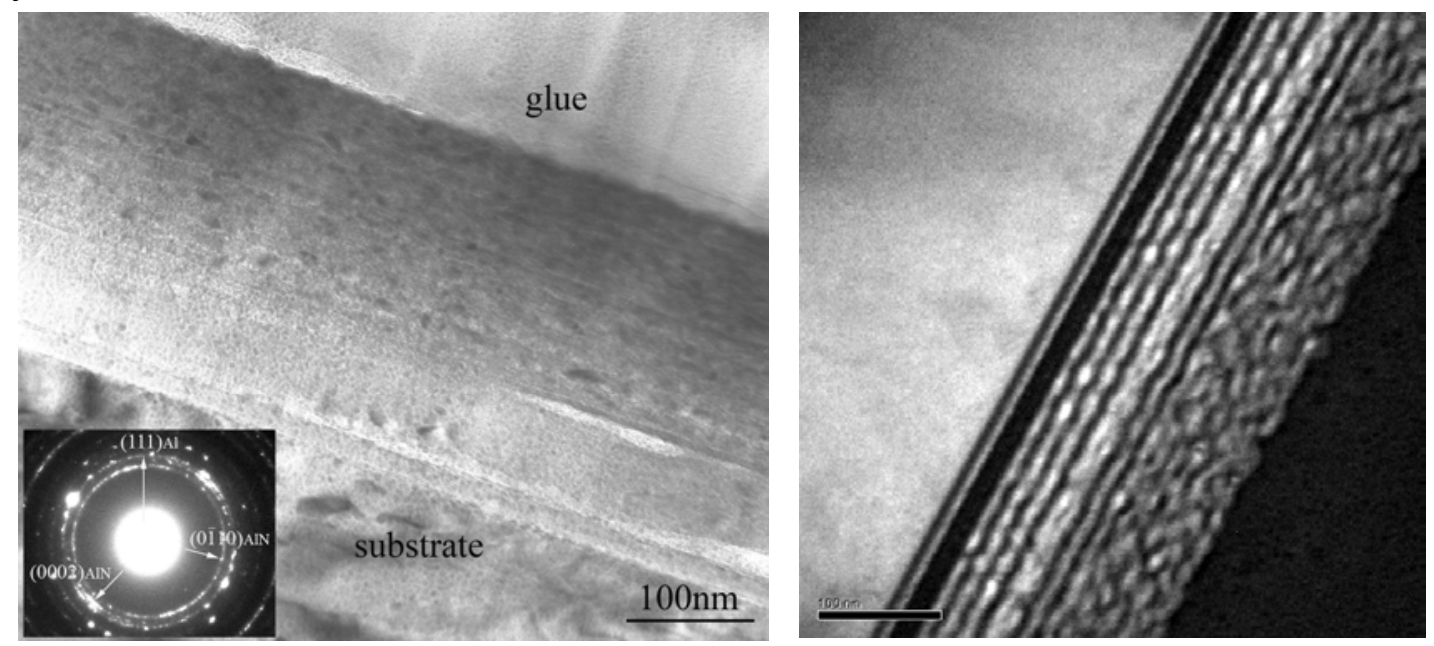

FIG. 2 : (left) A X-TEM image and diffraction pattern of a Al/AlN multilayer with a nominal bilayer thickness of $15 \mathrm{~nm}$. Also shown (right) is the corresponding Al map collected using the Al plasmon peak at $15 \mathrm{eV}$. 